\title{
Overwhelming research and clinical evidence of exercise medicine efficacy in cancer management-translation into practice is the challenge before us
}

\author{
R.U. Newton PhD*
}

The paper "Connecting people with cancer to physical activity and exercise programs: a pathway to create accessibility and engagement" 1 is very timely. As the authors have convincingly demonstrated, rigorous clinical and research evidence support something of which most medical and allied health professionals are cognizant: the benefit of physical activity and exercise for people with cancer. However, there is perhaps less recognition that targeted exercise medicine forms an essential component of overall patient management. It has been established that appropriate exercise undoubtedly provides benefit in terms of quality of life, physical function, and structure, and that it is likely to contribute to reduced morbidity and mortality, including cancer-specific mortality. But, there is a premise that simply performing any physical activity is sufficient to "tick this box," even though it has been established that the mode, dose, and volume of physical activity and exercise result in vastly different outcomes for the patient ${ }^{2,3}$.

The emphasis in the pathway model, in which patients transition back and forth between self-managed physical activity and exercise, public exercise programs, cancer-exercise programs, and specialized exercise medicine or rehabilitation programs, is very important and should be adopted as a foundation in the implementation of the pathway. Further, research is now homing in on the specifics of the "optimal" exercise prescription for the spectrum of health conditions and severities faced by cancer patients, such that a basic tenet of tailored exercise medicine that must be delivered within a flexible model is being established. An important point is that a physically active lifestyle should be encouraged in addition to a structured exercise regimen. It is also critical that the patient undertake targeted exercise while in therapy as medicine to ameliorate treatment-related adverse effects and to potentially enhance treatment effectiveness.

The challenge then is to actually facilitate patients not only to maintain recommended levels of physical activity, but also to engage in exercise programs that will optimally benefit their well-being and survival. The evidence highlighted in the paper by Santa Mina and colleagues is clear that cancer patients are not meeting the recommendations, which is substantially compromising quality of life, causing greater discomfort and physical and psychological distress, and actually reducing the opportunity for survival.

The pathway presented by the authors is likely to be a critical turning point in establishing a sustainable and effective model for increasing physical activity and targeted exercise for people with cancer. A key component of the pathway is the qualified exercise professional (QEP). The development of a workforce and growth in capacity in terms of the number of qualified individuals and facilities supporting implementation of quality exercise programs will require considerable expansion. A circuit breaker is the lack of financial support for the employment of, and service delivery by, QEPS. Currently, the benefit to the patient-and the associated reduction in overall health care and community costs - is not valued within the public and private systems. Whether funding comes through government health and medical systems, private health insurance, or a user-pay model, that issue has to be addressed. There is now solid health economics research demonstrating the value of QEPS in terms of both patient health and reduction in cost. That research must now be leveraged into government policy change ${ }^{4}$.

Another challenge to successful implementation of the pathway is that many QEPS will have modest training and experience in exercise oncology, given that their university degrees require broad knowledge and skills covering all chronic diseases and acute conditions. Post-bachelor qualifications or professional development specifically in exercise prescription and assessment for people with cancer could therefore quickly up-skill these underutilized allied health professionals to contribute substantially to the pathway.

The point about more aptly referring to home-based exercise as independent unsupervised exercise is important, because exercise in the home might not be appropriate because of concerns for safety and effectiveness. As highlighted in the paper, compared with home-based exercise programming, facility-based programs elicit much better physical and psychological benefits. The low cost and convenience of home-based exercise is recognized and 
could seem attractive at a superficial level; however, much work has to be done to explore supportive systems that can make such exercise settings safe and beneficial. It is likely that the optimal model will incorporate a combination of both self-managed and facility-based exercise programs. Given the established health and economic advantages when cancer patients meet physical activity guidelines and complete targeted exercise medicine prescription, government and private-sector investment in building appropriate exercise facilities for people with cancer or the incorporation of cancer-specific capability into existing facilities seems warranted.

Despite the large and growing research evidence and clinical experience showing that exercise medicine confers considerable benefit to people with cancer, a perception remains that patients, in particular those undergoing difficult treatments or having more advanced disease, should avoid physical activity and, in particular, strenuous exercise. That perception should be continually and rigorously challenged, and the relevant research evidence should be presented and incorporated within the university training of medical and allied health professionals. The authors provide strong arguments for the importance of patient education about physical activity and exercise and references to some excellent resources. However, along with the strategy, an education program must be established for the general population about the safety and efficacy of exercise for people with cancer so that not only patients, but also their friends and family understand and promote exercise for the person with cancer. Although considerable media interest in exercise medicine for people with cancer has been elicited, researchers and clinicians should continue to pursue opportunities for media coverage of their activities and of real-world examples of patient experiences.

I agree wholeheartedly with the statement by the authors that most cancer patients and survivors are not aware of, or are not able to access, exercise-related services and support. Exercise guidelines on "what to do" are extensive, but little is known about "how best to do it." Effective and widespread implementation is the challenge that must be addressed. Santa Mina et al. provide a well-researched and supported pathway to increase the engagement of cancer patients in physical activity and targeted exercise programs. Moving forward, it will be important to thoroughly research and evaluate the use of the pathway resources and the effect on exercise participation and physical activity levels in people with cancer, including their health outcomes. In addition, the financial cost-benefit must be rigorously evaluated if government, philanthropic, and corporate support of the pathway is to be attained, and if the required medical and allied health professionals and facilities are to be engaged.

\section{CONFLICT OF INTEREST DISCLOSURES}

I have read and understood Current Oncology's policy on disclosing conflicts of interest, and I declare that I have none.

\section{AUTHOR AFFILIATIONS}

*Exercise Medicine Research Institute, Edith Cowan University, Perth, Western Australia.

\section{REFERENCES}

1. Santa Mina D, Sabiston CM, Au D, et al. Connecting people with cancer to physical activity and exercise programs: a pathway to create accessibility and engagement. Curr Oncol 2018;25:149-62.

2. Friedenreich CM, Wang Q, Neilson HK, KopciukKA, McGregor SE, Courneya KS. Physical activity and survival after prostate cancer. Eur Urol 2016;70:576-85.

3. Hardee JP, Porter RR, Sui X, et al. The effect of resistance exercise on all-cause mortality in cancer survivors. Mayo Clin Proc 2014;89:1108-15.

4. Deloitte Access Economics and Exercise and Sports Science Australia (ESSA). The Value of Accredited Exercise Physiologists to Consumers in Australia. Ascot, Australia: EssA; 2016. 